\title{
SPANS IN LEBESGUE AND UNIFORM SPACES OF TRANSLATIONS OF STEP FUNCTIONS
}

\author{
RALPH PALMER AGNEW
}

1. Introduction. For each $p \geqq 1$, let $L_{p}$ be the Lebesgue space whose elements are real or complex valued measurable functions $f(x)$, defined over $-\infty<x<\infty$, for which the integral

$$
\int_{-\infty}^{\infty}|f(x)|^{p} d x
$$

is finite. The distance $\left\|f_{2}-f_{1}\right\|$ between two elements $f_{1}$ and $f_{2}$ of the space is defined by

$$
\left\|f_{2}-f_{1}\right\|=\left\{\int_{-\infty}^{\infty}\left|f_{2}(x)-f_{1}(x)\right|^{p} d x\right\}^{1 / p} .
$$

For each $p \geqq 1, L_{p}$ is a linear metric complete separable space.

Let $E$ be a set in $L_{p}$. The linear manifold $M(E)$ determined by $E$ is the set of all linear combinations (finite) of elements of $E$. The span $S_{p}(E)$ of $E$ in $L_{p}$ is the closure in $L_{p}$ of $M(E)$; an element $\phi$ of $L_{p}$ belongs to $S_{p}(E)$ if and only if to each $\epsilon>0$ corresponds an element $f_{\epsilon}$ of $M(E)$ such that $\left\|\phi-f_{\epsilon}\right\|<\epsilon$.

Let $f \in L_{p}$. For each real $h$, the translation $f(x+h)$ of $f(x)$ is also in $L_{p}$. Let $T_{f}$ denote the set of translations of $f$. Wiener $[2, \mathrm{pp} .7-9]^{1}$ showed that if $f \in L_{2}$, then $S_{2}\left(T_{f}\right)$ is the whole space $L_{2}$ if and only if the real zeros of the Fourier transform of $f$ form a set of measure 0 . He [2, pp. 9-19] showed also (and this was much more difficult) that if $f \in L_{1}$, then $S_{1}\left(T_{f}\right)$ is the whole space $L_{1}$ if and only if the Fourier transform of $f$ has no real zeros. He [2, p. 93] raised the question whether similar propositions hold for other values of $p$ and expressed a "suspicion" that they do, at least when $1 \leqq p \leqq 2$.

In view of the similar suspicions held by Wiener and others, a result recently announced by Segal [1] is surprising. Segal has shown that if $1<p<2$, then there is an element $f$ of $L_{p}$ such that (i) the zeros of the Fourier transform of $f$ form a set of measure 0 and (ii) the span $S_{p}\left(T_{f}\right)$ of the translations of $f$ does not include the whole space $L_{p}$.

This development will doubtless create interest in the search for criteria for $S_{p}\left(T_{f}\right)=L_{p}$. With the hope that both the result and the

Presented to the Society, November 24, 1944; received by the editors September 25, 1944.

${ }^{1}$ Numbers in brackets refer to the Bibliography at the end of the paper. 
special direct proof may throw light on the problem, the author presents the following result which he obtained ten years ago and has not previously announced.

THEOREM 1.3. If $p>1$ and $F$ is a simple step function, then $S_{p}\left(T_{F}\right)=L_{p}$.

2. Simple step functions. A simple step function is a function $F(x)$ which has a nonzero constant value over a finite interval $a \leqq x<a+2 b$ and is zero elsewhere. The positive constant $2 b$ is the width of the step function. With each simple step function $F$ of width $2 b$, we may associate the normal step function $G$ defined by

$$
G(x)=\left\{\begin{array}{l}
1,-b \leqq x<b, \\
0, \text { elsewhere }
\end{array}\right.
$$

Clearly, $M\left(T_{F}\right)=M\left(T_{G}\right)$ and $S_{p}\left(T_{F}\right)=S_{p}\left(T_{G}\right)$.

The Fourier transform $g(u)$ of the function $G(x)$ in (2.1) is

$$
g(u)=(2 \pi)^{-1 / 2} \int_{-\infty}^{\infty} G(x) e^{i u x} d x=(2 / \pi)^{1 / 2} \frac{\sin b u}{u}, \quad u \neq 0,
$$

and $g(0)=(2 / \pi)^{1 / 2} b$. This transform is bounded, continuous, and in $L_{p}$ for each $p>1$. Since $g(u)$ has real zeros, the second theorem of Wiener cited above implies that $S_{1}\left(T_{G}\right)$ is not the whole space $L_{1}$. Since the set of zeros has measure 0 , Wiener's first theorem implies that $S_{2}\left(T_{G}\right)=L_{2}$. Hence, when $p=2$, the conclusion of Theorem 1.3 follows from Wiener's theory.

3. Two lemmas. We shall use the two following lemmas.

Lemma 3.1. Let $p \geqq 1$ and let $f$ be an element of $L_{p}$. If $S_{p}\left(T_{f}\right)$ contains $g$, then $S_{p}\left(T_{f}\right)$ contains $S_{p}\left(T_{o}\right)$.

This is a consequence of the fact that linear combinations of translations of linear combinations of translations of $f$ are linear combinations of translations of $f$.

LEMMA 3.2. Let $p \geqq 1$ and let $F$ be an element of $L_{p}$. If $S_{p}\left(T_{F}\right)$ contains, for each $h>0$, a simple step function of width $h$, then $S_{p}\left(T_{F}\right)=L_{p}$.

Let $\phi$ be an element of $L_{p}$. Let $\epsilon>0$. A fundamental theorem on approximation gives a step function $g(x)$, a linear combination of simple step functions, such that $\|\phi-g\|<\epsilon$. Since $g$ is a linear combination of elements of $S_{p}\left(T_{F}\right), g$ itself is an element of $S_{p}\left(T_{F}\right)$. This establishes Lemma 3.2. 
4. Proof of Theorem 1.3. Using Lemma 3.2, we see that Theorem 1.3 is a consequence of the following lemma.

LEMMA 4.1. Let $p>1$. If $F$ is a simple step function, then $S_{p}\left(T_{F}\right)$ contains, for each $h>0$, a simple step function of width $h$.

Let $c, a$, and $h_{1}$ be the constants such that $F(x)=c$ when $a \leqq x<a+h_{1}$ and $F(x)=0$ otherwise. Let $h>0$, and let $g(x)$ be the simple step function for which $g(x)=1$ when $0 \leqq x<h$ and $g(x)=0$ otherwise. Let $\epsilon>0$. We shall prove Lemma 4.1 by giving an explicit formula for a linear combination $g_{\epsilon}(x)$ of translations of $F(x)$ such that $\left\|g-g_{\epsilon}\right\|<\epsilon$.

Let $m$ be the least integer for which $m h_{1}>h$ and let $H=m h_{1}$. Let

$$
G(x)=\sum_{j=0}^{m-1} c^{-1} F\left(x-j h_{1}-a\right) .
$$

Then

$$
G(x)=1
$$

$0 \leqq x<H$,

and $G(x)=0$ otherwise. Let $n$ be the least positive integer such that $(n H+h)^{1 / p}(2 n+1)^{-1}<\epsilon$. Let

$$
\begin{aligned}
g_{\epsilon}(x)=\sum_{k=0}^{n-1} & {\left[\left(1-\frac{2 k+1}{2 n+1}\right) G(x-k H)\right.} \\
& \left.-\left(1-\frac{2 k+2}{2 n+1}\right) G(x-k H-h)\right] .
\end{aligned}
$$

An understanding of the manner in which $g_{\epsilon}(x)$ approximates $g(x)$ is enhanced by drawing graphs of the terms of the sum in (4.4) and of the function $g_{\epsilon}(x)$. The function $g_{\epsilon}(x)$ is so constructed that

$$
\left|g(x)-g_{\epsilon}(x)\right|=(2 n+1)^{-1}, \quad 0 \leqq x<n H+h,
$$

and $\left|g(x)-g_{\epsilon}(x)\right|=0$ elsewhere. Hence

$$
\begin{aligned}
\int_{-\infty}^{\infty}\left|g(x)-g_{\epsilon}(x)\right| p d x & =\int_{0}^{n H+h}(2 n+1)^{-p} d x \\
& =(n H+h)(2 n+1)^{-p}
\end{aligned}
$$

and

$$
\left\|g-g_{\epsilon}\right\|=(n H+h)^{1 / p}(2 n+1)^{-1}<\epsilon .
$$

This completes the proof of Lemma 4.1 and hence also that of Theorem 1.3.

It is apparent that the above method for approximating $g(x)$ in $L_{p}$, 
$p>1$, fails when $p=1$; when $p=1$ and $\epsilon$ is small, one is unable to choose a positive integer $n$ such that the last inequality in (4.7) holds. As a matter of fact, no method can serve. For if $g(x)$ could be approximated in $L_{1}$ by linear combinations of translations of $G(x)$, then we could prove that $S_{1}\left(T_{G}\right)=L_{1}$; and this would contradict the second Wiener theorem cited in $\$ 1$.

When $h, H$, and $\epsilon$ are fixed, $\epsilon$ being small, the number $n$ of terms in (4.4) required to define the function $g_{\epsilon}(x)$ is a function of $p$ which increases as $p$ decreases. This indicates that the approximation in $L_{p}$ of $g(x)$ by linear combinations of translations of $G(x)$ becomes more difficult as $p$ decreases toward 1. This is in accord with the fact that the approximation is impossible when $p=1$.

5. An indicator. Let $p \geqq 1$ and let $f$ be an element of $L_{p}$. The number $I^{(p)}(f)$ defined by

$$
I^{(p)}(f)=\underset{g}{\text { l.u.b. }} \underset{F}{\text { g.l.b. }}\|g-F\|,
$$

where the g.l.b. is taken for all $F$ in $M\left(T_{f}\right)$ and the l.u.b. is then taken for all $g$ in $L_{p}$ for which $\|g\|=1$, is an indicator of the extent to which linear combinations of translations of $f(x) \operatorname{span} L_{p}$. If $S_{p}\left(T_{f}\right)=L_{p}$, then $I^{(p)}(f)=0$, and the converse holds. In any case, $0 \leqq I^{(p)}(f) \leqq 1$. It would be interesting to have information about $I^{(p)}(f)$. The author has very little. It seems to be true that if $f$ is a simple step function, then $I^{(1)}(f)=1$.

6. A corollary of Theorem 1.3. When one is seeking to determine whether $S_{p}\left(T_{f}\right)=L_{p}$, the following theorem may be helpful.

THEOREM 6.1. Let $p>1$, and let $f$ be an element of $L_{p}$. In order that $S_{p}\left(T_{f}\right)=L_{p}$, it is necessary and sufficient that $S_{p}\left(T_{f}\right)$ contain at least one simple step function.

Necessity is trival. Sufficiency is a consequence of Theorem 1.3 and Lemma 3.1.

7. Uniform approximation. The theorems of this section deal with uniform approximation, over the infinite interval $-\infty<x<\infty$, of functions $g(x)$ by linear combinations of translations of a given simple step function.

THEOREM 7.1. If $F(x)$ is a simple step function, then to each simple step function $g(x)$ and positive number $\epsilon$ corresponds a linear combination $g_{\epsilon}(x)$ of translations of $F(x)$ such that

$$
\left|g(x)-g_{\epsilon}(x)\right|<\epsilon, \quad-\infty<x<\infty .
$$


Let $\epsilon>0$. In case $g(x)=1$ over $0 \leqq x<h$ and $g(x)=0$ otherwise, (4.5) shows that the function $g_{\epsilon}(x)$ defined explicitly in (4.4) has the required property provided $n$ is chosen so great that $n^{-1}<\epsilon$. In case $g(x)=c$ over $a \leqq x<a+h$, the function $c g_{\epsilon}(x-a)$ has the required property. This proves Theorem 7.1.

THEOREM 7.3. Let $F(x)$ be a simple step function. Then to each continuous function $f(x)$, for which $f(x) \rightarrow 0$ as $x \rightarrow \infty$ and as $x \rightarrow-\infty$, and positive number $\epsilon$ corresponds a linear combination $f_{\epsilon}(x)$ of translations of $F(x)$ such that

$$
\left|f(x)-f_{\epsilon}(x)\right|<\epsilon, \quad-\infty<x<\infty .
$$

To prove this theorem, let $\epsilon>0$ and choose $M$ such that

$$
|f(x)|<\epsilon / 2, \quad|x| \geqq M .
$$

Because $f(x)$ is uniformly continuous over $-M \leqq x \leqq M$, we can construct a step function $\phi(x)$, vanishing outside the interval $-M \leqq x$ $\leqq M$, such that

$$
|f(x)-\phi(x)|<\epsilon / 2, \quad-\infty<x<\infty .
$$

Since $\phi(x)$ is a linear combination of simple step functions, it follows from Theorem 7.1 that there is a linear combination $f_{\epsilon}(x)$ of translations of $F(x)$ such that

$$
\left|\phi(x)-f_{\epsilon}(x)\right|<\epsilon / 2, \quad-\infty<x<\infty .
$$

The required representation (7.2) follows.

There are, of course, bounded functions which cannot be uniformly approximated by step functions, that is, by finite linear combinations of simple step functions. For example, $\sin x$ and $\sin x^{-1}$ (the latter function being defined to be 0 when $x=0$ ) cannot be. However, the conclusion of Theorem 7.3 holds for all functions $f(x)$ so approximable.

\section{BIBLIOGRAPHY}

1. I. E. Segal, The span of the translations of a function in a Lebesgue space, Proc. Nat. Acad. Sci. U. S. A. vol. 30 (1944) pp. 165-169.

2. N. Wiener, Tauberian theorems, Ann. of Math. (2) vol. 33 (1932) pp. 1-100.

CORNELl UNIVERSITY 